\title{
Bounding the symbol length in the Galois cohomology of function fields of $p$-adic curves
}

Venapally Suresh

Dedicated to my teacher Professor R. Parimala on her 60th birthday

\begin{abstract}
Let $K$ be a function field of a $p$-adic curve and $l$ a prime not equal to $p$. Assume that $K$ contains a primitive $l^{\text {th }}$ root of unity. We show that every element in the $l$-torsion subgroup of the Brauer group of $K$ is a tensor product of two cyclic algebras over $K$.
\end{abstract}

Mathematics Subject Classification (2000). Galois cohomology, central simple algebras, cyclic algebras, symbols.

Keywords. Primary 12G05; Secondary 11R58, 11R34.

\section{Introduction}

Let $k$ be a field and $l$ a prime number not equal to the characteristic of $k$. Let $\mu_{l}$ be the group of $l^{\text {th }}$ roots of unity and $\mu_{l}(m)$ the tensor product of $m$ copies of $\mu_{l}$. For $n \geq 0$, let $H^{n}\left(k, \mu_{l}\right)$ denote the $n^{\text {th }}$ Galois cohomology group with coefficients in $\mu_{l}$. Let $k^{*}=k \backslash\{0\}$. We have an isomorphism $k^{*} / k^{*^{l}} \rightarrow H^{1}\left(k, \mu_{l}\right)$. For $a \in k^{*}$, let $(a)$ denote its image in $H^{1}\left(k, \mu_{l}\right)$. For $a_{1}, \ldots, a_{m} \in k^{*}$, the cup product gives an element $\left(a_{1}\right) \cdot\left(a_{2}\right) \ldots\left(a_{m}\right) \in H^{n}\left(k, \mu_{l}(m)\right)$, which we call a symbol.

Assume that $k$ contains a primitive $l^{\text {th }}$ root of unity. Fix a primitive $l^{\text {th }}$ root of unity $\zeta \in k$. Then we have isomorphisms $\mu_{l} \rightarrow \mu_{l}(m)$ of Galois groups. Hence we have isomorphisms $H^{n}\left(k, \mu_{l}(m)\right) \rightarrow H^{n}\left(k, \mu_{l}\right)$. A symbol in $H^{n}\left(k, \mu_{l}\right)$ is simply the image of a symbol under this map.

A classical theorem of Merkurjev ([M]) asserts that every element in $H^{2}\left(k, \mu_{2}\right)$ is a sum of symbols. A deep result of Merkurjev and Suslin ([MS]) says that every element in $H^{2}\left(k, \mu_{l}\right)$ is a sum of symbols. By a theorem of Voevodsky ([V]), every element in $H^{n}\left(k, \mu_{2}\right)$ is a sum of symbols. Suppose that $k$ is a $p$-adic field. Local class field theory tells us that every element in $H^{2}\left(k, \mu_{l}\right)$ is a symbol and $H^{n}\left(k, \mu_{l}\right)=0$ for $n \geq 3$. If $k$ is a global field, then the global class field theory asserts that every element in $H^{n}\left(k, \mu_{l}\right)$ is a symbol. 
Question 1. Do there exist integers $N_{l}(n)(k)$ such that every element in $H^{n}\left(k, \mu_{l}\right)$ is a sum of at most $N_{l}(n)(k)$ symbols?

Of course, the answer to the above question is negative in general. It can be shown that for $K=k\left(X_{1}, \ldots, X_{n}, \ldots\right)$, there is no such $N_{l}(n)(K)$ for $n \geq 2$. However we can restrict to some special fields. It is well-known that if $N_{l}(n)(k)$ exist for $k$, then $N_{l}(n)(k((t)))$ exist. We ask the following

Question 2. Suppose that $N_{l}(n)(k)$ exist for some field $k$. Do they exist for $k(t)$ ?

This is an open question. However we can restrict to fields of arithmetic interest. For example we consider the $p$-adic fields. The most important result in this direction is the following

Theorem (Saltman, [S1], (cf. [S2])). Let $k$ be a p-adic field and $K / k(t)$ be a finite extension. Suppose that $l \neq p$. If $A$ is a central simple algebra over $K$ representing an element in $H^{2}\left(K, \mu_{l}\right)$, then ind $(A)$ divides $l^{2}$.

Let $K$ be as in the above theorem. Suppose $p \neq 2$. Let $\alpha \in H^{2}\left(K, \mu_{2}\right)$ and $A$ a central simple algebra over $K$ representing $\alpha$. Then by the above theorem, we have $\operatorname{ind}(A)=1,2,4$. If $\operatorname{ind}(A)=1$, then $\alpha$ is a trivial element. If $\operatorname{ind}(A)=2$, then it is well known that $\alpha$ is a symbol. Assume that $\operatorname{ind}(A)=4$. By a classical theorem of Albert ([A]), $\alpha$ is a sum of two symbols. For $H^{3}\left(K, \mu_{l}\right)$, we have the following

Theorem ([PS2], 3.5, (cf. [PS1], 3.9)). Let $k$ be a p-adic field and $K / k(t)$ be a finite extension. Suppose that $l \neq p$. Every element in $H^{3}\left(K, \mu_{l}\right)$ is a symbol.

Let $k$ and $K$ be as above. The field $K$ is of cohomological dimension 3 and $H^{n}\left(K, \mu_{l}\right)=0$ for $n \geq 4$. By the above theorem, $N_{l}(3)(K)=1$ and the only case where $N_{l}(n)(K)$ is to be determined is for $n=2$. It is known that $N_{l}(2)(K) \geq 2$ (cf. [S1], Appendix). In this article we prove the following

Theorem. Let $k$ be a $p$-adic field and $K / k(t)$ be a finite extension. Suppose that $l \neq p$. Every element in $H^{2}\left(K, \mu_{l}\right)$ is a sum of at most two symbols; in other words, $N_{l}(2)(K)=2$.

\section{Some preliminaries}

In this section we recall a few basic facts about Galois cohomology groups and divisors on arithmetic surfaces. We refer the reader to ([C]), ([Li1]), ([Li2]) and ([Se]). 
Let $k$ be a field and $l$ a prime number not equal to the characteristic of $k$. Assume that $k$ contains a primitive $l^{\text {th }}$ root of unity. Let $\zeta \in k$ be a primitive $l^{\text {th }}$ root of unity. Let $\mu_{l}$ be the group of $l^{\text {th }}$ roots of unity. Since $k$ contains a primitive $l^{\text {th }}$ root of unity, the absolute Galois group of $k$ acts trivially on $\mu_{l}$. For $m \geq 1$, let $\mu_{l}(m)$ denote the tensor product of $m$ copies of $\mu_{l}$. By fixing a primitive $l^{\text {th }}$ root of unity $\zeta$ in $k$, we have isomorphisms of Galois modules $\mu_{l}(m) \rightarrow \mu_{l}$. Throughout this paper we fix a primitive $l^{\text {th }}$ root of unity and identify $\mu_{l}(m)$ with $\mu_{l}$.

Let $H^{n}(k, A)$ be the $n^{\text {th }}$ Galois cohomology group of the absolute Galois group $\Gamma$ of $k$ with values in a discrete $\Gamma$-module $A$. The identification of $\mu_{l}(m)$ with $\mu_{l}$ gives an identification of $H^{n}\left(k, \mu_{l}(m)\right)$ with $H^{n}\left(k, \mu_{l}\right)$. In the rest of this paper we use this identification.

Let $k^{*}=k \backslash\{0\}$. For $a, b, c \in k^{*}$ we have the following relations in $H^{2}\left(k, \mu_{l}\right)$.

(1) $(a) \cdot(b c)=(a) \cdot(b)+(a) \cdot(c)$;

(2) $(a) \cdot(b)=-((b) \cdot(a))$;

(3) $(a) \cdot\left(b^{l}\right)=0$;

(4) $(a) \cdot(-a)=0$.

If $l \geq 3$, we have $(a) \cdot(a)=(a) \cdot\left((-1)^{l} a\right)=(a) \cdot(-a)=0$.

Let $K$ be a field and $l$ a prime number not equal to the characteristic of $K$. Let $v$ be a discrete valuation of $K$. The residue field of $v$ is denoted by $\kappa(v)$. Suppose $\operatorname{char}(\kappa(v)) \neq l$. Then there is a residue homomorphism $\partial_{v}: H^{n}\left(K, \mu_{l}(m)\right) \rightarrow$ $H^{n-1}\left(\kappa(v), \mu_{l}(m-1)\right)$. Let $\alpha \in H^{n}\left(K, \mu_{l}(m)\right)$. We say that $\alpha$ is unramified at $v$ if $\partial_{v}(\alpha)=0$; otherwise it is said to be ramified at $v$.

Let $\mathcal{X}$ be a regular integral scheme of dimension $d$, with function field $K$. Let $\mathcal{X}^{1}$ be the set of points of $\mathcal{X}$ of codimension 1 . A point $x \in \mathcal{X}^{1}$ gives rise to a discrete valuation $v_{x}$ on $K$. The residue field of this discrete valuation ring is denoted by $\kappa(x)$. The corresponding residue homomorphism is denoted by $\partial_{x}$. We say that an element $\zeta \in H^{n}\left(K, \mu_{l}(m)\right)$ is unramified at $x$ if $\partial_{x}(\zeta)=0$; otherwise it is said to be ramified at $x$. We define the ramification divisor $\operatorname{ram} x(\zeta)=\sum x$ as $x$ runs over points in $\mathcal{X}^{1}$ where $\zeta$ is ramified. Suppose $C$ is an irreducible subscheme of $\mathcal{X}$ of codimension 1. Then the generic point $x$ of $C$ belongs to $X^{1}$ and we set $\partial_{C}=\partial_{x}$. If $\alpha \in H^{n}\left(K, \mu_{l}(m)\right)$ is unramified at $x$, then we say that $\alpha$ is unramified at $C$. We say that $\alpha$ is unramified on $\mathcal{X}$ if it is unramified at every point of $\mathcal{X}^{1}$.

Let $k$ be a $p$-adic field and $K$ the function field of a smooth projective geometrically integral curve $X$ over $k$. By the resolution of singularities for surfaces (cf. [Li1] and [Li2]), there exists a regular projective model $\mathcal{X}$ of $X$ over the ring of integers $\mathcal{O}_{k}$ of $k$. We call such an $\mathcal{X}$ a regular projective model of $K$. Since the generic fibre $X$ of $X$ is geometrically integral, it follows that the special fibre $\bar{X}$ is connected. Further if $D$ is a divisor on $\mathcal{X}$, there exists a proper birational morphism $\mathcal{X}^{\prime} \rightarrow \mathcal{X}$ such that the total transform of $D$ on $\mathcal{X}^{\prime}$ is a divisor with normal crossings (cf. [Sh], Theorem, p. 38 and Remark 2, p. 43). We use this result throughout this paper without further 
reference. If $P \in \mathcal{X}$ is a closed point and $f \in K$ is a unit at $P$, then we denote the image of $f$ in the residue field at $P$ by $f(P)$.

Let $k$ be a $p$-adic field and $K$ the function field of a smooth projective geometrically integral curve over $k$. Let $l$ be a prime number not equal to $p$. Assume that $k$ contains a primitive $l^{\text {th }}$ root of unity. Let $\alpha \in H^{2}\left(K, \mu_{l}\right)$. Let $\mathcal{X}$ be a regular projective model of $K$ such that $\operatorname{ram}_{\mathcal{X}}(\alpha)=C+E$, where $C$ and $E$ are regular curves with normal crossings. We have the following

Theorem 1.1 (Saltman [S1]). Let $K, \alpha, \mathcal{X}, C$ and $E$ be as above and $P \in C \cup E$. Let $R$ be the local ring at $P$. Let $\pi, \delta \in R$ be local equations of $C$ and $E$ respectively at $P$.

(1) If $P \in C \backslash E$ (or $E \backslash C$ ), then $\alpha=\alpha^{\prime}+(\pi) \cdot(u)$ (or $\alpha=\alpha^{\prime}+(\delta) \cdot(u)$ ) for some unit $u \in R, \alpha^{\prime} \in H^{2}\left(K, \mu_{l}\right)$ unramified on $R$.

(2) If $P \in C \cap E$, then either $\alpha=\alpha^{\prime}+(\pi) \cdot(u)+(\delta) \cdot(v)$ or $\alpha=\alpha^{\prime}+(\pi) \cdot\left(u \delta^{i}\right)$ for some units $u, v \in R, \alpha^{\prime} \in H^{2}\left(K, \mu_{l}\right)$ unramified on $R$.

Let $P \in C \cap E$. Suppose that $\alpha=\alpha^{\prime}+(\pi) \cdot(u)+(\delta) \cdot(v)$ for some units $u, v \in R, \alpha^{\prime} \in H^{2}\left(K, \mu_{l}\right)$ unramified on $R$ and $\pi, \delta$ are local equations of $C$ and $E$ respectively. Then $u(P)=\partial_{C}(\alpha)(P)$ and $v(P)=\partial_{E}(\alpha)(P)$. Note that $u(P)$ and $v(P)$ are uniquely defined modulo $l^{\text {th }}$ powers. Following Saltman ([S3], §2), we say that $P$ is a hot point of $\alpha$ if $u(P)$ and $v(P)$ do not generate the same subgroup of $\kappa(P)^{*} / \kappa(P)^{*^{l}}$.

We have the following

Theorem 1.2 (Saltman ([S3], 5.2). Let $k, K, \alpha, \mathcal{X}$ be as above. Then $\alpha$ is a symbol if and only if there are no hot points of $\alpha$.

\section{The main theorem}

Let $k$ be a $p$-adic field and $K / k(t)$ be a finite extension. Let $l \geq 3$ be a prime number not equal to $p$. Assume that $k$ contains a primitive $l^{\text {th }}$ root of unity. Let $\beta \in H^{2}\left(K, \mu_{l}\right)$ and $\mathcal{X}$ a regular proper model of $K$. Let $\phi: X^{\prime} \rightarrow \mathcal{X}$ be a blow-up such that $X^{\prime}$ is a regular proper model of $K$ and $\operatorname{ram}_{X^{\prime}}(\beta)=C^{\prime}+E^{\prime}$, where $C^{\prime}$ and $E^{\prime}$ are two regular curves with normal crossings (cf. $\S 1$ or [S1], Proof of 2.1). Let $Q \in C^{\prime} \cap E^{\prime}$. Let $C_{1}^{\prime} \subset C^{\prime}$ and $E_{1}^{\prime} \subset E^{\prime}$ be the irreducible curves containing $Q$. Let $R^{\prime}=\mathcal{O}_{X^{\prime}, Q}$ be the regular local ring at $Q$ and $m_{Q}$ its maximal ideal. We have $m_{Q}=\left(\pi^{\prime}, \delta^{\prime}\right)$, where $\pi^{\prime}$ and $\delta^{\prime}$ are local equations of $C_{1}^{\prime}$ and $E_{1}^{\prime}$ at $Q$ respectively. Let $v_{C_{1}^{\prime}}$ and $v_{E_{1}^{\prime}}$ be the discrete valuations on $K$ at $C_{1}^{\prime}$ and $E_{1}^{\prime}$ respectively.

Let $P=\phi(Q)$. Let $R$ be the regular local ring at $P$ and $m_{P}$ its maximal ideal. We have the induced homomorphism $\phi^{*}: R \rightarrow R^{\prime}$ of local rings, which is injective. Let $\pi, \delta \in R$ be such that $m_{P}=(\pi, \delta)$. 
Lemma 2.1. Suppose that $\beta=\beta^{\prime}+\left(f^{\prime}\right) \cdot\left(g^{\prime}\right)$ for some $f^{\prime}, g^{\prime} \in K$ and $\beta^{\prime}$ unramified on $R^{\prime}$. Then $Q$ is not a hot point of $\beta$.

Proof. Since $\beta^{\prime}$ is unramified on $R^{\prime}$, the ramification data of $\beta$ on $R^{\prime}$ is same as that of $\left(f^{\prime}\right) \cdot\left(g^{\prime}\right)$. Since $\left(f^{\prime}\right) \cdot\left(g^{\prime}\right)$, being a symbol, has no hot points ([S3], cf. 1.2), $Q$ is not a hot point of $\beta$.

Lemma 2.2. Suppose that $\beta=\beta^{\prime}+(\delta) \cdot(g v)+(f) \cdot(g)$, where $\beta^{\prime}$ is unramified on $R^{\prime}, f \in R$ is not divisible by $\delta$ and $v, g \in R$ are units with $g(P)=v(P)$. Then $Q$ is not a hot point of $\beta$.

Proof. We have $m_{Q}=\left(\pi^{\prime}, \delta^{\prime}\right)$ and $\beta$ has ramification on $R^{\prime}$ only at $\pi^{\prime}$ and $\delta^{\prime}$. Since $R / m_{P} \hookrightarrow R^{\prime} / m_{Q}$, we have $g(Q)=g(P)=v(P)=v(Q)$. If $C_{1}^{\prime}$ (or $\left.E_{1}^{\prime}\right)$ is the strict transform of a curve on $X$, then either $\delta$ is a local equation of $C_{1}^{\prime}$ or $v_{C_{1}^{\prime}}(\delta)=0$. In fact, if $C_{1}^{\prime}$ is the strict transform of $C_{1}$ on $\mathcal{X}$, then $v_{C_{1}}(\delta)=v_{C_{1}^{\prime}}(\delta)$ and $\delta$ itself being a prime in $R$, the assertion follows.

Suppose that $C_{1}^{\prime}$ and $E_{1}^{\prime}$ are strict transforms of two irreducible curves on $\mathcal{X}$. If $\delta$ is not a local equation for either $C_{1}^{\prime}$ or $E_{1}^{\prime}$, we claim that $(\delta) \cdot(g v)$ is unramified on $R^{\prime}$. In fact, since $g$ and $v$ are units in $R,(\delta) \cdot(g v)$ is unramified on $R$ except possibly at $\delta$. Since $f$ is not divisible by $\delta,(f) \cdot(g)$ is unramified at $\delta$. Since $\beta$ is ramified on $R^{\prime}$ only at $\pi^{\prime}$ and $\delta^{\prime}$ and $\delta$ is not one of them, $(\delta) \cdot(g v)$ is unramified on $R^{\prime}$. By (2.1), $Q$ is not a hot point of $\beta$. Assume that $\delta$ is a local equation for one of them, say $C_{1}^{\prime}$. Since $\delta$ does not divide $f$, we have $\partial_{C_{1}^{\prime}}(\beta)=\overline{v g}$ and $\partial_{E_{1}^{\prime}}(\beta)=\tilde{g}^{v_{E_{1}^{\prime}}}(f)$, where bar denotes the image in the residue field of $C_{1}^{\prime}$ and tilde denotes the image in the residue field of $E_{1}^{\prime}$. Since $\beta$ is ramified at $E_{1}^{\prime}, v_{E_{1}^{\prime}}(f)$ is not a multiple of $l$. We have $\partial_{C_{1}^{\prime}}(\beta)(Q)=v(Q) g(Q)=g(Q)^{2}$ and $\partial_{E_{1}^{\prime}}(\beta)(Q)=g(Q)^{v_{E_{1}^{\prime}}(f)}$. Since $l \neq 2$ and $v_{E_{1}^{\prime}}(f)$ is not a multiple of $l, g(Q)^{2}$ and $g(Q)^{v_{E_{1}^{\prime}}(f)}$ generate the same subgroup modulo $l^{\text {th }}$ powers. Hence $Q$ is not a hot point of $\beta$.

Suppose that $C_{1}^{\prime}$ is a strict transform of an irreducible curve on $X$ and $E_{1}^{\prime}$ is an exceptional curve on $\mathcal{X}^{\prime}$. We have $\partial_{E_{1}^{\prime}}(\beta)=(\tilde{g v})^{v_{E_{1}^{\prime}}(\delta)} \tilde{g}^{v_{E_{1}^{\prime}}(f)}$. Since $E_{1}^{\prime}$ is an exceptional fibre in $X^{\prime}$, the residue field of $R$ is contained in the residue field at $E_{1}^{\prime}$. Hence $\partial_{E_{1}^{\prime}}(\beta)=(g(P) v(P))^{v_{E_{1}^{\prime}}(\delta)} g(P)^{v_{E_{1}^{\prime}}(f)}=g(P)^{2 v_{E_{1}^{\prime}}(\delta)+v_{E_{1}^{\prime}}(f)}$. Since $\beta$ is ramified at $E_{1}^{\prime}, 2 v_{E_{1}^{\prime}}(\delta)+v_{E_{1}^{\prime}}(f)$ is not a multiple of $l$. Suppose $\delta$ is a local equation of $C_{1}^{\prime}$ at $Q$. Since $\delta$ does not divide $f$ and $\nu_{C_{1}^{\prime}}(\delta)=1$, we have $\partial_{C_{1}^{\prime}}(\beta)=\overline{g v}$. Thus $\partial_{C_{1}^{\prime}}(\beta)(Q)=g(P) v(P)=g(P)^{2}$. Since $l \neq 2$ and $2 v_{E_{1}^{\prime}}(\delta)+v_{E_{1}^{\prime}}(f)$ is not a multiple of $l$, the subgroups generated by $g(Q)^{2}$ and $g(P)^{2 v_{E_{1}^{\prime}}(\delta)+v_{E_{1}^{\prime}}(f)}$ are equal modulo $l^{\text {th }}$ powers. Hence $Q$ is not a hot point of $\beta$. Suppose $\delta$ is not a local equation of $C_{1}^{\prime}$ at $Q$. We have $\partial_{C_{1}^{\prime}}(\beta)=\bar{g}^{\nu_{C_{1}^{\prime}}(f)}$. Since $\beta$ is ramified at $C_{1}^{\prime}, v_{C_{1}^{\prime}}(f)$ is not a multiple of $l$. Thus as above $Q$ is not a hot point of $\beta$. 
The case $E_{1}^{\prime}$ is a strict transform of an irreducible curve on $\mathcal{X}$ and $C_{1}^{\prime}$ is an exceptional curve in $X^{\prime}$ follows on similar lines.

Suppose that both $C_{1}^{\prime}$ and $E_{1}^{\prime}$ are exceptional curves in $X^{\prime}$. Then as above we have $\partial_{C_{1}^{\prime}}(\beta)=g(P)^{2 v} C_{1}^{\prime}(\delta)+v_{C_{1}^{\prime}}(f)$ and $\partial_{E_{1}^{\prime}}(\beta)=g(P)^{2 v_{E_{1}^{\prime}}(\delta)+v_{E_{1}^{\prime}}(f)}$. Since $\beta$ is ramified at $C_{1}^{\prime}$ and $E_{1}^{\prime}, 2 v_{C_{1}^{\prime}}(\delta)+v_{C_{1}^{\prime}}(f)$ and $2 v_{E_{1}^{\prime}}(\delta)+v_{E_{1}^{\prime}}(f)$ are not multiples of $l$. In particular, the subgroups generated by $g(P)^{2 v_{C_{1}^{\prime}}(\delta)+v_{C_{1}^{\prime}}(f)}$ and $g(P)^{2 v_{E_{1}^{\prime}}(\delta)+v_{E_{1}^{\prime}}(f)}$ are equal modulo the $l^{\text {th }}$ powers. Thus $Q$ is not a hot point of $\beta$.

Lemma 2.3. Suppose that $\beta=\beta^{\prime}+(\pi) \cdot(u)+(\delta) \cdot(v)$, where $\beta^{\prime}$ unramified on $R^{\prime}$ and $u, v \in R$ units with $u(P)=v(P)$. Then $Q$ is not a hot point of $\beta$.

Proof. Since $\beta$ is ramified at $C_{1}^{\prime}$, either $v_{C_{1}^{\prime}}(\pi)$ or $v_{C_{1}^{\prime}}(\delta)$ is not divisible by $l$. In particular their sum $v_{C_{1}^{\prime}}(\pi \delta)$ is non-zero. We have

$$
\partial_{C_{1}^{\prime}}(\beta)(Q)=u(P)^{v_{C_{1}^{\prime}}(\pi)} v(P)^{v_{C_{1}^{\prime}}(\delta)}=u(P)^{v_{C_{1}^{\prime}}(\pi \delta)} .
$$

Suppose that $v_{C_{1}^{\prime}}(\pi \delta)$ is a multiple of $l$. Since $v_{C_{1}}^{\prime}(\pi \delta)$ is non-zero, $C_{1}^{\prime}$ is an exceptional curve. As in the proof of $(2.2)$, we see that $\partial_{C_{1}^{\prime}}(\beta)=u(P)^{v_{1}{ }^{\prime}}{ }^{(\pi \delta)}=1$. Which is a contradiction, as $\beta$ is ramified at $C_{1}^{\prime}$. Hence $v_{C_{1}^{\prime}}(\pi \delta)$ is not a multiple of l. Similarly, we have $\partial_{E_{1}^{\prime}}(\beta)(Q)=u(P)^{v_{E_{1}^{\prime}}(\pi \delta)}$ and $v_{E_{1}^{\prime}}(\pi \delta)$ is not a multiple of $l$. Hence $u(P)^{v_{1}{ }_{1}(\pi \delta)}$ and $u(P)^{v_{E_{1}^{\prime}}(\pi \delta)}$ generate the same subgroup of $\kappa(P)^{*}$ modulo $\kappa(P)^{*^{l}}$ and $Q$ is not a hot point of $\beta$.

Theorem 2.4. Let $k$ be a $p$-adic field and $K / k(t)$ be a finite extension. Let $l$ be a prime number not equal to $p$. Suppose that $k$ contains a primitive $l^{\text {th }}$ root of unity. Then every element in $H^{2}\left(K, \mu_{l}\right)$ is a sum of at most two symbols.

Proof. If $l=2$, then, as we mentioned before, by ([A]), $\alpha$ is a sum of at most two symbols. Assume that $l \geq 3$. Let $\alpha \in H^{2}\left(K, \mu_{l}\right)$. Let $\mathcal{X}$ be a regular proper model of $K$ such that $\operatorname{ram}_{\mathcal{X}}(\alpha)=C+E$, where $C$ and $E$ are regular curves with normal crossings.

Let $P \in C \cup E$ be a closed point of $\mathcal{X}$. Let $R_{P}$ be the regular local ring at $P$ on $X$ and $m_{P}$ be its maximal ideal.

Let $T$ be a finite set of closed points of $\mathcal{X}$ containing $C \cap E$ and at least one closed point from each irreducible curve in $C$ and $E$. Let $A$ be the semi-local ring at $T$ on $\mathcal{X}$. Let $\pi_{1}, \ldots \pi_{r}, \delta_{1}, \ldots, \delta_{s} \in A$ be prime elements corresponding to irreducible curves in $C$ and $E$ respectively. Let $f_{1}=\pi_{1} \ldots \pi_{r} \delta_{1} \ldots \delta_{s} \in A$. Let $P \in C \cap E$. Then $P \in C_{i} \cap E_{j}$ for unique irreducible curves $C_{i}$ in $C$ and $E_{j}$ in $E$. Then $\pi=\pi_{i}$ and $\delta=\delta_{j}$ are local equations of $C$ and $E$ at $P$. We have $\alpha=\alpha^{\prime}+(\pi) \cdot\left(u_{P}\right)+(\delta) \cdot\left(v_{P}\right)$ 
or $\alpha=\alpha^{\prime}+(\pi) \cdot\left(u_{P} \delta^{i}\right)$ for some units, $u_{P}, v_{P} \in R$ and $\alpha^{\prime}$ unramified on $R$ ([S1], cf. 1.1). By the choice of $f_{1}$, we have $f_{1}=\pi \delta w_{P}$ for some $w_{P} \in A$ which is a unit at $P$. Let $u \in A$ be such that $u(P)=w_{P}(P)^{-1} u_{P}(P)$ for all $P \in C \cap E$. Let $f=f_{1} u \in A$. Then, we have $(f)=C+E+F$, where $F$ is a divisor on $\mathcal{X}$ which avoids $C, E$ and all the points of $C \cap E$. Further, for each $P \in C_{i} \cap E_{j}$, we have $f=\pi_{i} \delta_{j} w_{i j}$ for some $w_{i j} \in A$ such that $w_{i j}(P)=u_{P}(P)$.

By a similar argument, choose $g \in K$ satisfying

(1) $(g)=C+G$, where $G$ is a divisor on $\mathcal{X}$ which avoids $C, E, F$ and also avoids the points of $C \cap E, C \cap F, E \cap F$;

(2) if $P \in E \cap F$ and $\alpha=\alpha^{\prime}+(\delta) \cdot(v)$ for some unit $v \in R_{P}$ and $\alpha^{\prime}$ is unramified at $P$, then $g(P)=v(P)$.

Since $C \cap E \cap F=\emptyset$, such a $g$ exists.

We claim that $\beta=\alpha+(f) \cdot(g)$ is a symbol.

Let $\phi: X^{\prime} \rightarrow \mathcal{X}$ be a blow up of $\mathcal{X}$ such that $\mathcal{X}^{\prime}$ is a regular proper model of $K$ and $\operatorname{ram}_{X^{\prime}}(\beta)=C^{\prime}+E^{\prime}$, where $C^{\prime}$ and $E^{\prime}$ are regular curves with normal crossings.

To show that $\beta$ is a symbol, it is enough to show that $\beta$ has no hot points ([S3], cf. 1.2). Let $Q \in C^{\prime} \cap E^{\prime}$. Let $P=\phi(Q)$. Then $P$ is a closed point of $\mathcal{X}$, $R=\mathcal{O}_{X, P} \subset \mathcal{O}_{X^{\prime}, Q}=R^{\prime}$ and the maximal ideal $m_{P}$ of $R$ is contained in the maximal ideal $m_{Q}$ of $R^{\prime}$. Let $m_{Q}=\left(\pi^{\prime}, \delta^{\prime}\right)$, with $\pi^{\prime}$ and $\delta^{\prime}$ be local equations of $C^{\prime}$ and $E^{\prime}$ at $Q$ respectively.

Suppose that $P \notin C \cup E$. Then $\alpha$ is unramified at $P$ and hence unramified at $Q$. By (2.1), $Q$ is not a hot point of $\beta$.

Assume that $P \in C \cup E$.

Suppose that $P \in C \cap E$. Let $\pi$ and $\delta$ be local equations of $C$ and $E$ at $P$ respectively. Then $m_{P}=(\pi, \delta)$. By the choice of $f$ and $g$, we have $f=\pi \delta w_{1}$ and $g=\pi w_{2}$ for some units $w_{1}, w_{2} \in R$. In particular, $\beta$ is ramified on $R$ only at $\pi$ and $\delta$. Suppose that $\alpha=\alpha^{\prime}+(\pi) \cdot(u)+(\delta) \cdot(v)$ for some units $u, v \in R$ and $\alpha^{\prime}$ unramified on $R$. We have

$$
\begin{aligned}
\beta & =\alpha+(f) \cdot(g) \\
& =\alpha^{\prime}+(\pi) \cdot(u)+(\delta) \cdot(v)+\left(\pi \delta w_{1}\right) \cdot\left(\pi w_{2}\right) \\
& =\alpha^{\prime}+(\pi) \cdot(u)+\left(\delta w_{1}\right) \cdot(v)+\left(w_{1}^{-1}\right) \cdot(v)+(\pi) \cdot\left(\pi w_{2}\right)+\left(\delta w_{1}\right) \cdot\left(\pi w_{2}\right) \\
& =\alpha^{\prime}+\left(w_{1}^{-1}\right) \cdot(v)+(\pi) \cdot\left(u \pi w_{2}\right)+\left(\delta w_{1}\right) \cdot\left(\pi w_{2} v\right) \\
& =\alpha^{\prime}+\left(w_{1}^{-1}\right) \cdot(v)+(\pi) \cdot\left(u w_{2}\right)+\left(\delta w_{1}\right) \cdot\left(\pi w_{2} v\right) \\
& =\alpha^{\prime}+\left(w_{1}^{-1}\right) \cdot(v)+\left(\pi w_{2} v\right) \cdot\left(u w_{2}\right)+\left(w_{2}^{-1} v^{-1}\right) \cdot\left(u w_{2}\right)+\left(\delta w_{1}\right) \cdot\left(\pi w_{2} v\right) \\
& =\alpha^{\prime}+\left(w_{1}^{-1}\right) \cdot(v)+\left(w_{2}^{-1} v^{-1}\right) \cdot\left(u w_{2}\right)+\left(\pi w_{2} v\right) \cdot\left(u w_{2} \delta^{-1} w_{1}^{-1}\right) .
\end{aligned}
$$

Since $\alpha^{\prime}+\left(w_{1}^{-1}\right) \cdot(v)+\left(w_{2}^{-1} v^{-1}\right) \cdot\left(u w_{2}\right)$ is unramified on $R$, by (2.1), $Q$ is not a hot point of $\beta$. 
Suppose that $\alpha=\alpha^{\prime}+(\pi) \cdot\left(u \delta^{i}\right)$ for some units, $u, v \in R$ and $\alpha^{\prime}$ unramified on $R$. Then we have

$$
\begin{aligned}
\beta & =\alpha+(f) \cdot(g) \\
& =\alpha^{\prime}+(\pi) \cdot\left(u \delta^{i}\right)+\left(\pi \delta w_{1}\right) \cdot\left(\pi w_{2}\right) \\
& =\alpha^{\prime}+(\pi) \cdot\left(u \delta^{i}\right)+\left(\delta w_{1} w_{2}^{-1}\right) \cdot\left(\pi w_{2}\right) \\
& =\alpha^{\prime}+(\pi) \cdot\left(u \delta^{i}\left(\delta w_{1} w_{2}^{-1}\right)^{-1}\right)+\left(\delta w_{1} w_{2}^{-1}\right) \cdot\left(w_{2}\right) \\
& =\alpha^{\prime}+(\pi) \cdot\left(\delta^{i-1} u w_{1}^{-1} w_{2}\right)+\left(\delta w_{1} w_{2}^{-1}\right) \cdot\left(w_{2}\right) .
\end{aligned}
$$

If $i=1$, then $\beta=\alpha^{\prime}+(\pi) \cdot\left(u w_{1}^{-1} w_{2}\right)+\left(\delta w_{1} w_{2}^{-1}\right) \cdot\left(w_{2}\right)$. Since, by the choice of $f, u(P)=w_{1}(P)$, by (2.3), $Q$ is not a hot point of $\beta$. Assume that $i>1$. Then $1 \leq i-1<l-1$. Let $i^{\prime}$ be the inverse of $1-i$ modulo $l$. We have

$$
\begin{aligned}
\beta= & \alpha^{\prime}+(\pi) \cdot\left(\delta^{i-1} u w_{1}^{-1} w_{2}\right)+\left(\delta w_{1} w_{2}^{-1}\right) \cdot\left(w_{2}\right) \\
= & \alpha^{\prime}+\left(\delta^{1-i} u^{-1} w_{1} w_{2}^{-1}\right) \cdot(\pi)+\left(\delta w_{1} w_{2}^{-1}\right) \cdot\left(w_{2}\right) \\
=\alpha^{\prime} & +\left(\left(\delta\left(u^{-1} w_{1} w_{2}^{-1}\right)^{i^{\prime}}\right)^{1-i}\right) \cdot(\pi)+\left(\delta\left(u^{-1} w_{1} w_{2}^{-1}\right)^{i^{\prime}}\right) \cdot\left(w_{2}\right) \\
& \quad+\left(\left(u^{-1} w_{1} w_{2}^{-1}\right)^{-i^{\prime}}\right) \cdot\left(w_{2}\right)+\left(w_{1} w_{2}^{-1}\right) \cdot\left(w_{2}\right) \\
=\alpha^{\prime} & +\left(\left(u^{-1} w_{1} w_{2}^{-1}\right)^{-i^{\prime}}\right) \cdot\left(w_{2}\right)+\left(w_{1} w_{2}^{-1}\right) \cdot\left(w_{2}\right) \\
& \quad+\left(\left(\delta\left(u^{-1} w_{1} w_{2}^{-1}\right)^{i^{\prime}}\right)^{1-i}\right) \cdot\left(\pi^{1-i} w_{2}\right) .
\end{aligned}
$$

Since $\alpha^{\prime}+\left(\left(u^{-1} w_{1} w_{2}^{-1}\right)^{-i^{\prime}}\right) \cdot\left(w_{2}\right)+\left(w_{1} w_{2}^{-1}\right) \cdot\left(w_{2}\right)$ is unramified on $R$, by $(2.1)$, $Q$ is not a hot point of $\beta$.

Suppose that $P \in C \backslash E$. We have $\alpha=\alpha^{\prime}+(\pi) \cdot(u)$ for some unit $u$ in $R$ and $\alpha^{\prime}$ unramified on $R$. We also have $f=\pi f_{1}$ for some $f_{1} \in R$ which is not divisible by $\pi$. We have

$$
\begin{aligned}
\beta & =\alpha+(f) \cdot(g) \\
& =\alpha^{\prime}+(\pi) \cdot(u)+\left(\pi f_{1}\right) \cdot(g) \\
& =\alpha^{\prime}+\left(f_{1}^{-1}\right) \cdot(u)+\left(\pi f_{1}\right) \cdot(u)+\left(\pi f_{1}\right) \cdot(g) \\
& =\alpha^{\prime}+\left(f_{1}^{-1}\right) \cdot(u)+\left(\pi f_{1}\right) \cdot(g u) .
\end{aligned}
$$

If $f_{1}$ is a unit in $R$, then $\alpha^{\prime}+\left(f_{1}^{-1}\right) \cdot(u)$ is unramified on $R$, by (2.1), $Q$ is not a hot point of $\beta$. Assume that $f_{1}$ is not a unit in $R$. Then $P \in C \cap F$ and $g=\pi g_{1}$ for some unit $g_{1} \in R$. We have

$$
\begin{aligned}
\beta & =\alpha+(f) \cdot(g) \\
& =\alpha^{\prime}+(\pi) \cdot(u)+\left(\pi f_{1}\right) \cdot\left(\pi g_{1}\right) \\
& =\alpha^{\prime}+\left(\pi g_{1}\right) \cdot(u)+\left(g_{1}^{-1}\right) \cdot(u)+\left(\pi f_{1}\right) \cdot\left(\pi g_{1}\right) \\
& =\alpha^{\prime}+\left(g_{1}^{-1}\right) \cdot(u)+\left(\pi g_{1}\right) \cdot\left(u\left(\pi f_{1}\right)^{-1}\right) .
\end{aligned}
$$


Since $\alpha^{\prime}+\left(g_{1}^{-1}\right) \cdot(u)$ is unramified on $R$, by (2.1), $Q$ is not a hot point of $\beta$.

Suppose that $P \in E \backslash C$. Then $\alpha=\alpha^{\prime}+(\delta) \cdot(v)$ for some unit $v \in R$ and $f=\delta f_{1}$ for some $f_{1} \in R$ which is not divisible by $\delta$. Suppose that $f_{1}$ is a unit in $R$. Then, as above, $Q$ is not a hot point of $\beta$. Assume that $f_{1}$ is not a unit in $R$. Then $P \in E \cap F$ and $g$ is a unit in $R$. We have

$$
\begin{aligned}
\beta & =\alpha+(f) \cdot(g) \\
& =\alpha^{\prime}+(\delta) \cdot(v)+\left(\delta f_{1}\right) \cdot(g) \\
& =\alpha^{\prime}+(\delta) \cdot(v g)+\left(f_{1}\right) \cdot(g) .
\end{aligned}
$$

Since $\alpha^{\prime}$ is unramified on $R$ and by the choice of $g, g(P)=v(P)$, by (2.2), $Q$ is not a hot point of $\beta$.

By ([S3], cf. 1.2), $\beta$ is symbol. Thus $\alpha=(f) \cdot(g)-\beta$ is a sum of at most two symbols.

Acknowledgments. We would like to thank for support from UGC (India) under the SAP program.

\section{References}

[A] A. A. Albert, Normal division algebras of degree four over an algebraic field. Trans. Amer. Math. Soc. 34 (1931), 363-372. Zbl 0004.10002 MR 1501642

[Ar] J. K. Arason, Cohomologische Invarianten quadratischer Formen. J. Algebra 36 (1975), 448-491. Zbl 0314.12104 MR 0389761

[AEJ] J. K. Arason, R. Elman, and B. Jacob, Fields of cohomological 2-dimension three. Math. Ann. 274 (1986), 649-657. Zbl 0576.12025 MR 0848510

[C] J.-L. Colliot-Thèlène, Birational invariants, purity, and the Gersten conjecture. In $K$-theory and algebraic geometry: connections with quadratic forms and division algebras, Proc. Sympos. Pure Math. 58, Part 1, Amer. Math. Soc., Providence, RI, 1995, 1-64. Zbl 0834.14009 MR 1327280

[Li1] J. Lipman, Introduction to resolution of singularities. In Algebraic geometry, Proc. Sympos. Pure Math. 29, Amer. Math. Soc., Providence, R.I., 1975, 187-230. Zbl 0306.14007 MR 0389901

[Li2] Lipman, J., Desingularization of two-dimensional schemes. Ann. of Math. 107 (1978), 151-207. Zbl 0349.14004 MR 0491722

[M] A. S. Merkurjev, On the norm residue symbol of degree 2. Dokl. Akad. Nauk. SSSR 261 (1981), 542-547; English transl. Soviet Math. Dokl. 24 (1981), no. 3, 546-551. Zbl 0496.16020 MR 0638926

[MS] A. S. Merkurjev and A. A. Suslin, $K$-cohomology of Severi-Brauer varieties and the norm residue homomorphism. Dokl. Akad. Nauk SSSR 264 (1982), 555-559; English transl. Soviet Math. Dokl. 25 (1982), no. 3, 690-693. Zbl 0525.18007 MR 0659762 
[PS1] R. Parimala and V. Suresh, Isotropy of quadratic forms over function fields in one variable over $p$-adic fields. Inst. Hautes Études Sci. Publ. Math. 88 (1998), 129-150. Zbl 0972.11020 MR 1733328

[PS2] R. Parimala and V. Suresh, The $u$-invariant of the function fields of $p$-adic curves. Preprint 2007. arXiv:0708.3128v1

[S1] D. J. Saltman, Division Algebras over p-adic curves. J. Ramanujan Math. Soc. 12 (1997), 25-47. Zbl 0902.16021 MR 1462850

[S2] D. J. Saltman, Correction to "Division algebras over $p$-adic curves". J. Ramanujan Math. Soc. 13 (1998), 125-130. Zbl 0920.16008 MR 1666362

[S3] D. J. Saltman, Cyclic algebras over p-adic curves. J. Algebra 314 (2007), 817-843. Zbl 1129.16014 MR 2344586

[Se] J.-P. Serre, Cohomologie Galoisienne: progrès et problèmes. Astérisque 227 (1995), Exp. No. 783, 229-257. Zbl 0837.12003 MR 1321649

[Sh] I. R. Shafarevich, Lectures on minimal models and birational transformations of two dimensional schemes. Tata Inst. Fund. Res. Lect. Math. Phys. 37, Tata Institute of Fundamental Research, Bombay 1966. Zbl 164.51704 MR 0217068

[V] V. Voevodsky, Motivic cohomology with Z/2-coefficients. Inst. Hautes Études Sci. Publ. Math. 98 (2003), 59-104. Zbl 1057.14028 MR 2031199

Received April 1, 2008

Venapally Suresh, Department of Mathematics and Statistics, University of Hyderabad, Gachibowli, Hyderabad - 500046, India

E-mail:vssm@uohyd.ernet.in 